\title{
Expression of BRD4 in patients with nonvalvular atrial fibrillation and its role in postoperative recurrence and the development of atrial fibrosis
}

\author{
de Liu ${ }^{1}$, Long Wang ${ }^{1}$, Wei $\mathrm{Li}^{1}$, Wei Zhou ${ }^{2}$, and Haiyan Zhou ${ }^{2}$ \\ ${ }^{1}$ Guizhou Medical University \\ ${ }^{2}$ The Affiliated Hospital of Guizhou Medical University
}

January 25, 2022

\begin{abstract}
Objective: Through clinical studies and animal experiments, we explored the expression of BRD4 in patients with NVAF and its role in the postoperative recurrence and occurrence of atrial fibrosis. Methods: 74 patients with NVAF and 73 patients with PSVT were included. The BRD4 levels of the patients were measured. Thirty-Six Sprague Dawley(SD) rats were randomly divided into three groups. The incidence and duration of AF in each group were recorded. Masson's trichrome staining was performed. Western blot was used to detect the expression of BRD4, TGF- $\beta 1$, P-Smad2/3, Smad2/3, Smad7, $\mathrm{Cx} 43$, and type III collagen in each group. Results: The BRD4 levels were significantly higher in patients with AF than those of PSVT,especially in patients with persistent $\mathrm{AF}$ and postoperative recurrence of AF and AF with left atrial low voltage $(P<$ 0.05). The collagen volume fraction and average induced rate of AF and average duration of AF and levels of BRD4, TGF- $\beta 1$, P-Smad2/3, Smad2/3, and type III collagen were significantly highest in the AF + CTL group than those of the CTL group and AF + JQ-1 group $(P<0.05)$. Conclusions: BRD4 was correlated with AF and atrial fibrosis. Inhibition of BRD4 expression significantly improved the degree of left atrial fibrosis in rats with $\mathrm{AF}$ and reduced the incidence of $\mathrm{AF}$ and the duration of AF. BRD4 may be involved in atrial fibrosis and remodeling through the TGF- $\beta 1 /$ Smad signaling pathway, thus participating in the occurrence and maintenance of AF.
\end{abstract}

\section{Introduction}

Atrial fibrillation (AF) is a common arrhythmia in clinical practice [1]. A survey by Zhang Shu et al. [2] on 19,368 natural patients aged 35 years old in different regions of China showed that the age-adjusted prevalence of $\mathrm{AF}$ was approximately $0.74 \%$. Globally, the current prevalence of AF in adults is estimated to be between $2 \%$ and $4 \%$, and is expected to increase [3-6]. AF represents a significant burden to patients, doctors, and the healthcare system. AF is an important cause of heart failure, ischemic stroke and other diseases, which seriously harm the health of humans. With the continued updating of diagnosis and treatment methods, the success rate of AF treatment is gradually improving, but it still does not reach the expected goal. Currently, the treatment of AF mainly includes drug control of ventricular rate, drug cardioversion and maintenance sinus rhythm, percutaneous radiofrequency catheter ablation, percutaneous cryoballoon ablation, and surgical Mazes. However, drug therapy has disadvantages of a low success rate and many side effects, while surgical Maze procedures cause significant trauma and carry a high risk of perioperative death.

Ablation is more effective than drugs [7-10] and has been recommended for AF in domestic and foreign guidelines [11, 12]. However, the long-term success rate of ablation for paroxysmal AF that has been followed up over 1 year is only $50 \%-80 \%$ [13-15], and is even lower for persistent AF [16]. Moreover, the degree of atrial fibrosis is more severe in patients with persistent AF than in patients with paroxymoron AF. Atrial fibrosis is closely related to the occurrence and maintenance of AF. Treatment of atrial fibrosis 
can effectively reduce the occurrence and burden of $\mathrm{AF}$, but the current clinical methods are limited and ineffective. Therefore, it is important to further study the mechanism of atrial fibrosis and its relationship with the occurrence and development of AF.

Bromodomain-containing protein 4 (BRD4) plays an important role in the process of fibrosis by regulating cell transcription and the cell cycle. Recent studies have found that BRD4 inhibitors can be used for treating fibrotic diseases via target gene-related pathways including the extracellular matrix (ECM) receptor interaction, adhesion protein, PDGF pathway, JNK/MAPK pathway, and the NF- $x \mathrm{~B}$ pathway. Therefore, BRD4 is considered a potential target in fibrosis. Studies have shown that the inhibition of BRD4 expression can reduce myocardial cell apoptosis and ventricular myocyte fibrosis [17-19]. In 2019, at the 40th Annual Meeting of the American Heart Association, a wall report study presented by Professor Yigang Li [20] found that BRD4 inhibition could reduce the induction rate of AF and alleviate atrial fibrosis, suggesting that BRD4 is a new target for treating atrial fibrosis and AF. This study was the first to establish a link between BRD4 and atrial fibrosis and AF. However, the correlation between the transforming growth factor- $\beta$ (TGF$\beta$ )/SMAD signaling pathway, which is closely related to AF and atrial fibrosis, with BRD4 has not yet been reported. Furthermore, there has been no clinical study on the expression of BRD4 in patients with AF in combination with non-valvular disease and non-ischemic cardiomyopathy. Additionally, the relationship between BRD4 and AF recurrence after ablation remains unknown.

Based on the above views, we further verified the association between BRD4 and AF and atrial fibrosis through clinical observation and animal experiments. As the pathogenesis of AF in valvular disease is more complex, the atrial fibrosis of nonvalvular atrial fibrillation (NVAF) is most commonly caused by valvular disease itself, and valve replacement is the basic treatment strategy. Valvular AF accounts for approximately $12.9 \%$ of patients with AF, and the subjects of the current study have NVAF.

\section{Methods}

Clinical study participants

This is a prospective, randomized, double-blind clinical study. The research subjects were patients with NVAF $(\mathrm{n}=74)$ or paroxysmal supraventricular tachycardia (PSVT, $\mathrm{n}=73$ ). All patients who met the indications in the guidelines for radiofrequency ablation of arrhythmias were selected, and all underwent radiofrequency ablation for the first time. We conducted a sub-group analysis to evaluate the associations between AF and BRD4. All participants provided written informed consent, and all protocols were performed in accordance with the Medical Science Ethics Committee of the Affiliated Hospital of Guizhou Medical University.

\section{Ascertainment of AF and PSVT}

AF was diagnosed by the ECG and/or dynamic ECG results before radiofrequency ablation. AF was defined according to the guidelines [12]. PSVT was diagnosed by the ECG and/or intracardiac electrophysiology results, and include atrioventricular node reentrant tachycardia (AVNRT) and atrioventricular reentrant tachycardia (AVRT).

Research groups and clinical indicators

Patients with AF were defined as the AF group; patients with paroxysmal supraventricular tachycardia were defined as the PSVT group; patients with AF with normal left atrial voltage were defined as the NLAV group; patients with AF with left atrial low-voltage areas (LAVs) were defined as the LAV group; patients with AF who maintained sinus rhythm after ablation were defined as the NreAF group; and patients with $\mathrm{AF}$ who had recurrence AF/atrial tachycardia/atrial flutter after ablation were defined as the reAF group.

For all enrolled patients, to detect BRD4 levels by qRT-PCR before radiofrequency ablation, $2 \mathrm{ml}$ of elbow venous blood was extracted in the morning after a state of abstinence from drinking and fasting for at least $8 \mathrm{~h}$. Transthoracic echocardiography was completed in parallel for all candidates prior to radiofrequency ablation, to measure the left atrium anterior and posterior diameter (LAAPD) and left ventricular ejection 
fraction (LVEF). For patients with NVAF, transesophageal echocardiography was completed in the $24 \mathrm{~h}$ prior to radiofrequency ablation to exclude left atrial and/or left atrial appendage thrombosis while measuring the left atrial appendage peak emptying velocity (LAA-PEV). For patients with NVAF, left atrial highdensity matrix mapping was performed to observe the bipolar voltage at each point in the left atrium during radiofrequency ablation. After ablation, all patients with $\mathrm{AF}$ underwent follow-up at 1, 3, 6, 12, and 18 months by standard 12-lead ECG and/or 72-h dynamic ECG.

BRD4 levels of human peripheral venous blood

We used qRT-PCR to detect the levels of BRD4 [21]. The following primers were used:BRD4:5'CCCTGAAGCCGTCCACACT-3' (forward) and 5'-TTCTCAGCTTGAGGTTTCCTTTTC-3' (reverse); GAPDH:5'-GATCCCTCCAAAATCAAGTGG-3' (forward) and 5'-GGAGGCATTGCTGATGATCT-3' (reverse). The $2^{-[?][?] \mathrm{Ct}}$ method was used to analyze the relative expression of target genes.

Cardiac parameters measured by echocardiography

A Philips iE33 color Doppler ultrasonic diagnostic instrument and quantitative analysis software QLab 8.1 were used to measure LAAPD,LVEF, and LAA-PEV. For transthoracic echocardiography, the twodimensional probe (S5-1) was selected and the frequency was adjusted to 2.0-3.5 MHz. The LAAPD was measured using the method of two-dimensional radial line through long axial section of the left ventricle parasternal, and the LVEF was calculated using the biplane Simpson method. For transesophageal echocardiography, the real-time three-dimensional transesophageal matrix probe (X7-2t) was selected and the frequency was adjusted to $2-7 \mathrm{MHz}$, while the LAA-PEV was measured by the pulsed wave doppler(PW) method. In patients with sinus rhythm, the flow spectrum of the left atrial appendage (LAA) was a regular bidirectional waveform:positive waveform was an empty wave, and negative waveform was a filling wave. The peak value of the positive waveform was recorded, and was named LAA-PEV (Fig. 1). In patients with $\mathrm{AF}$, the flow spectrum of the LAA was an irregular serrated waveform, and the peak value of its positive waveform was also selected as LAA-PEV (Fig. 2).

High density matrix mapping of the left atrium

Under sinus rhythm, the Abbott EnSite Velocity ${ }^{\mathrm{TM}} 5.0$ intelligent heart 3D mapping system was used to construct the left atrium 3D model with the assistance of EnSite Velocity ${ }^{\mathrm{TM}}$ body surface electrodes. The Inquiry $^{\mathrm{TM}}$ AFocus $\mathrm{II}^{\mathrm{TM}}$ 10-pole adjustable bending pulmonary vein catheter was used to detect the bipolar voltage at each point in the left atrium. The WorkMate ${ }^{\mathrm{TM}}$ Claris ${ }^{\mathrm{TM}}$ electrophysiological recording system was used to statistically analyze the local bipolar voltage at each point in the left atrium, and the overall average value of the bipolar voltage at each point in the left atrium was calculated. A local bipolar voltage $>0.5 \mathrm{mV}$ in the left atrium was defined as normal voltage, while a bipolar voltage $<0.5 \mathrm{mV}$ was defined as a low voltage area (Fig. 3 and Fig. 4). To facilitate recording and description, the left atrium was divided into anterior, apical, and posterior anatomical regions.

Follow-up after ablation

After ablation, all patients with AF completed follow-up at 1, 3, 6, 12, and 18 months by standard 12-lead ECG and/or 72-h dynamic ECG. Three months after ablation was defined as the "blank period." Recurrence of $\mathrm{AF}$ was defined as atrial flutter/AF/tachycardia with a duration $>30 \mathrm{~s}$, which was occurring more than 3 months after ablation. In this study, the time point for recurrence assessment was set at 12 months after ablation. If the follow-up time was less than 12 months, the actual follow-up time was recorded. All patients had a follow-up time of at least 6 months.

Animal experiments

All protocols were performed in accordance with the Animal Experiment Ethics Committee of Guizhou Medical University. Thirty-six male SD rats aged 6-8 weeks were equally assigned to three groups according to the principle of randomness and double blindness; the groups included the blank control group (CTL), AF model group (AF + CTL), and AF model + JQ-1 group (AF + JQ-1). SD rats in the AF + JQ-1 group 
were intravenously injected with (+)-JQ-1 for 3 weeks through the caudal vein; the dosage was $8 \mathrm{mg} / \mathrm{kg}$ and the frequency was once a week [19]. All of the SD rats underwent transesophageal electrophysiological examination under anesthesia. SD rats in the AF + CTL and AF + JQ-1 groups also underwent rapid atrial pacing to induce AF for 3 weeks. The ECG trace was continuously recorded by a biologic signal recording and analysis system which filtered out the signals below $10 \mathrm{~Hz}$ and above $100 \mathrm{~Hz}$.The AF inducibility

was performed by the transesophageal burst rapid pacing with a stimulating amplitude of 2-fold atrial capture threshold. Four consecutive bursts of rapid electrical stimulation for $30 \mathrm{~s}(20,30,40$, and $50 \mathrm{~Hz})$ were applied to induce AF with 3-minute pause[22]. AF was defined as an abnormal ECG showing rapid and fragmented $\mathrm{P}$ wave with absolute irregular RR intervals (the time elapsed between two successive R-waves of the QRS signal on the electrocardiogram) for at least 2 seconds, and AF could be induced at least two times of five sets of pacing[22-24] (Fig. 5). The mean incidence and duration of AF in each group were recorded. The mean incidence of AF of each rat was the number of times that AF was successfully induced divided by 5 , and the unit was percentage (\%). The mean duration of AF was the average of the sum of the duration of $\mathrm{AF}$ of each rat, and the unit of value was seconds (s).

After the above steps, the SD rats were anesthetized and killed, and the left atrium tissue samples were collected and marked for subsequent experiments. Six left atrium tissue samples were collected from each group, and fixed in $4 \%$ paraformaldehyde, embedded in paraffin, and cut into sections (5um thick). Sections of left atrium were stained by Masson's staining to detect collagen as previously reported[23-24]. The level of cardiac fibrosis was determined by the percentage of the fibrosis area to the total area (\% cardiac fibrosis, collagen volume fraction) using the ImageJ software[23-24].Six left atrium tissue samples were collected from each group, and western blot was used to detect the expression levels of BRD4, TGF- $\beta 1$, P-SMad2/3, SMAD2/3, Smad7, Cx43, and type III collagen in each group.

\section{Statistical analysis}

The participant characteristics are summarized as the mean standard deviation (SDs) or percentage and count. Non-normally distributed measurement data were represented by the median ( 25 to 75 percentiles) and the rank sum test was used for inter-group comparison. $\chi^{2}$ tests were used to examine the association between categorical variables and AF. Spearman correlation test was used for correlation analysis, and $t$ -tests or ANOVA were used for continuous variables. The test-level $a=0.05$, GraphPad Prism 5.0 was used to compile the graphs, and ImageJ software was used for gray value analysis. SPSS version 24.0 software was used for all statistical analyses. All probability values for statistical tests used $P<0.05$ as a statistically significant value and are two-tailed.

\section{Results}

\section{Participant characteristics}

Table 1 shows the characteristics of the participants with AF and PSVT. The mean values of sex, age, hypertension, diabetes, smoking, and heavy alcohol intake were not significantly different between participants with AF and those with PSVT.

\section{BRD4 levels, LAAPD, and LVEF in participants with AF and PSVT}

Table 2 shows the BRD4 levels of peripheral venous blood, LAAPD, and LVEF in patients with AF and PSVT. The $2^{-[?][?] \mathrm{Ct}}$ method was used to analyze the relative expression of BRD4 genes, and the obtained value is expressed by RQ value. The mean values of BRD4 levels and LAAPD were significantly higher in participants with AF than in those with PSVT. However, the mean values of LVEF were significantly lower in participants with AF than in those with PSVT.

BRD4 levels, LAAPD, LVEF, LAA-PEV, left atrial low voltage and postoperative recurrence in participants with paroxysmal $\mathrm{AF}$ and persistent $\mathrm{AF}$

Table 3 shows the BRD4 levels of peripheral venous blood, LAAPD, LVEF, LAA-PEV, left atrial low voltage, and postoperative recurrence in participants with paroxysmal $\mathrm{AF}$ and persistent $\mathrm{AF}$. The mean 
values of LVEF and LAA-PEV were significantly higher in participants with paroxysmal AF than in those with persistent AF. However, the mean BRD4 levels, LAAPD, left atrial low voltage, and postoperative recurrence were significantly lower in participants with paroxysmal AF than in those with persistent AF.

BRD4 levels, LAAPD, LVEF, LAA-PEV, and left atrial low voltage of participants in the NreAF and reAF groups

Table 4 shows the BRD4 levels of peripheral venous blood, LAAPD, LVEF, LAA-PEV, and left atrial low voltage of participants in the NreAF and reAF groups. The mean values of LVEF and LAA-PEV were significantly higher in participants in the NreAF group than those in the reAF group. However, the mean values of BRD4 levels, LAAPD, and left atrial low voltage were significantly lower in participants in the NreAF group than those in the reAF group.

BRD4 levels, LAAPD, LVEF, and LAA-PEV of the participants in the NLAV and LAV groups

Table 5 shows the BRD4 levels of peripheral venous blood, LAAPD, LVEF, and LAA-PEV of participants in the NLAV and LAV groups. The mean values of LVEF and LAA-PEV were significantly higher in participants in the NLAV group than those in the LAV group. However, the mean values of BRD4 levels and LAAPD were significantly lower in participants in the NLAV group than those in the LAV group.

The mean incidence and duration of AF in the SD rats in the AF + CTL group and AF + JQ-1 group

Table 6 shows the mean incidence and duration of AF in SD rats in the AF + CTL group and AF + JQ-1 group. The mean incidence and duration of AF were significantly higher in SD rats in the AF + CTL group than those in the AF + JQ-1 group.

Fibrosis of the left atrium evaluated by Masson's trichrome staining of SD rats in the CTL, AF + CTL, and $\mathrm{AF}+\mathrm{JQ}-1$ groups

Figure 6 shows the collagen volume fraction and left atrial fibrosis evaluated by Masson's trichrome staining of SD rats in the CTL group $(n=6), A F+$ CTL group $(n=6)$, and AF + JQ-1 group $(n=6)$. The mean collagen volume fraction of the left atrium was significantly higher in SD rats in the AF + CTL group than those in the AF + JQ-1 group and CTL group. The mean collagen volume fraction of the left atrium was significantly higher in SD rats in the AF + JQ-1 group than those in the CTL group (D). The area of fibrotic collagen (stained blue) was largest in the AF + CTL group (B), least in the CTL group (A), and the second largest in AF + JQ-1 group (C).

Fibrosis-related indicators detected by western blot(WB) of SD rats in the CTL, AF + CTL, and AF + JQ1 groups

Figure 7 shows the fibrosis-related indicators (BRD4, TGF- $\beta 1$, P-Smad2/3, Smad2/3, Smad7, CX43, and type III collagen) detected by WB of SD rats in the CTL group $(\mathrm{n}=6), \mathrm{AF}+\mathrm{CTL}$ group $(\mathrm{n}=6)$, and AF + JQ1 group $(\mathrm{n}=6)$. The mean values of the BRD4, TGF- $\beta 1$, P-Smad2/3, Smad2/3, and type III collagen were significantly higher in SD rats in the AF + CTL group than those in the AF + JQ1 and CTL groups. However, the mean values of the Smad7 and CX43 were significantly lower among SD rats in the AF + CTL group than among those in the AF + JQ1 and CTL groups.

\section{Discussion}

This study showed that BRD4 was positively correlated with atrial fibrosis, AF, postoperative recurrence of AF, LVAs, and LAAPD, and was negatively correlated with LVEF. BRD4 may be involved in atrial fibrosis and remodeling through the TGF- $\beta 1 /$ Smad signaling pathway, and thus participate in the occurrence and maintenance of AF.

Association between LAAPD and AF

Studies have shown that atrial muscle fibrosis is significantly aggravated in patients with AF, which was positively correlated with LAAPD [25]. Left atrial fibrosis and structural remodeling were more obvious in 
patients with AF with left atrial enlargement [26, 27]. Clinically, LAAPD is usually measured by echocardiography or cardiac magnetic resonance(CMR) to reflect the size of the left atrium. Only LAAPD has been widely used in large-scale clinical studies that could be reviewed. LAAPD can accurately estimate the left atrial volume through the nonlinear equation of the elliptic model [28]. Multiple studies have shown that LAAPD enlargement is an independent predictor of recurrence after AF ablation [29-31]. Our study showed that the mean values of LAAPD were significantly higher in participants of the AF group, patients with persistent $\mathrm{AF}$, and participants in the reAF group than those in the PSVT group, patients with paroxysmal $\mathrm{AF}$, and participants in the NreAF group, and all the differences were statistically significant. It has been suggested that the left atrial enlargement is more obvious in patients with AF, especially those with persistent AF. Moreover, patients with AF with left atrial enlargement had an increased rate of postoperative recurrence.

\section{Association between LAA-PEV and AF}

Some studies have found that LAA is one of the potential trigger points of spontaneous AF [32]. Increased LAA volume and decreased LAA ejection fraction were predictors of recurrence after ablation of AF [33-36]. The decrease in LAA-PEV was positively correlated with postoperative recurrence of AF [37]. Transesophageal echocardiography could accurately determine LAA-PEV in patients with AF. In this study, the LAA-PEV of patients in the reAF group was significantly lower than that of patients in the NreAF group, and the difference was statistically significant. This result suggests that patients with AF with reduced LAA-PEV had an increased postoperative recurrence rate.

Association between left atrial fibrosis and AF

Excessive synthesis and irregular deposition of extracellular matrix (ECM) proteins are thought to be involved in the onset and maintenance of AF [38]. These collagen-based scars (collagen type I and III) could form barriers that blocked electrical conduction and separated well-connected syncytes, thus directly interfering with electrical conduction among cardiomyocytes [39]. Additionally, fibroblasts and myofibroblasts could form cell membrane fusions with cardiomyocytes to form intercellular gap junctions via connexin 40, 43, and 45 ( $\mathrm{Cx} 40, \mathrm{Cx} 43$, and $\mathrm{Cx} 45)$ [40, 41]. These material bases, through changing the electrical conduction velocity among cardiac myocytes and increasing the electrical conduction heterogeneity, lead to the formation of complex electrical conduction reentry in the atrium, and ultimately lead to the occurrence and maintenance of AF [42-44]. Atrial remodeling is a key factor linking all the mechanisms associated with AF, and atrial fibrosis was the most prominent feature of atrial remodeling [45]. In the rat AF model, compared to the CTL group, the degree of left atrial fibrosis and collagen volume fraction in the AF + CTL group were significantly higher. Our results showed that compared to the CTL group, the level of type III collagen was significantly higher and the level of Cx43 was significantly lower in the AF + CTL group.

\section{Association between LVAs and AF}

$\mathrm{AF}$ and atrial fibrosis are cause and effect and promote each other. The presence of left atrial fibrosis increased the recurrence rate of AF after ablation. Cardiac MRI (CMR) is a well-established method for detecting left atrial fibrosis and can be used to noninvasively identify the scar matrix of the left atrium, which is known as the fibrotic area [46-48]. However, CMR was found to be inaccurate in evaluating left atrial fibrosis in patients with persistent $\operatorname{AF}[49,50]$. CMR and left atrial bipolar voltage mapping were used to detect left atrial fibrosis and left atrial low-voltage region (bipolar voltage $<0.5 \mathrm{mV}$ )/scar area (bipolar voltage $<0.05 \mathrm{mV}$ ) in participants with sinus rhythm, and the left atrial low voltage/scar area was highly consistent with the left atrial fibrosis area in CMR in Jeong's study [51]. Subsequent studies also confirmed that LVAs measured by left atrial bipolar voltage mapping were consistent with the left atrial fibrosis regions in CMR [52, 53]. Therefore, it is reasonable to use LVAs to represent left atrial fibrosis in clinical practice. Several studies had shown that the presence of LVAs is an independent predictor of postoperative AF recurrence [54-57]. Our results showed that the mean values of LVAs were significantly lower in participants with paroxysmal AF and those in the NreAF group than those with persistent AF and those in the reAF group. These results suggest that the decrease in left atrial voltage was more obvious 
in patients with persistent AF. Moreover, LAVs were positively correlated with postoperative recurrence of $\mathrm{AF}$.

Association among LAAPD, LVEF, and LAVs

The relationship among LAAPD, LVEF, and LAVs has not been reported previously. Studies have shown that left atrial fibrosis was more pronounced in patients with AF with enlarged left atrium. In patients with AF with reduced LVEF, the pressure of the left atrium was increased, which promoted left atrial enlargement and left atrial fibrosis. Therefore, it is reasonable to use LVAs to represent left atrial fibrosis in clinical practice. We speculated that LAAPD and LVEF were correlated with LAVs. The results of this study showed that the mean values of LAAPD were significantly lower in participants in the NLAV group than those in the LAV group. However, the mean values of LVEF were significantly higher in participants in the NLAV group than those in the LAV group. These findings suggest that LAAPD is positively correlated with LAVs, while LVEF is negatively correlated with LAVs.

Association between TGF- $\beta /$ Smads and AF

Previous studies have confirmed that TGF- $\beta$ is closely associated with the occurrence of tissue fibrosis [58] and plays a crucial role in the process of myocardial fibrosis. TGF- $\beta$ activates the synthesis of ECM protein through the Smads signal transduction pathway to cause excessive deposition of ECM and myocardial fibrosis. Atrial fibrosis affects the development of AF through the TGF- $\beta 1 /$ Smads pathway [59]. TGF- $\beta$ expression was mainly completed through TGF- $\beta /$ Smads signaling pathway. Multiple studies have shown that downregulation of the TGF- $\beta /$ Smads signaling pathway could decrease TGF- $\beta$ expression, attenuate the degree of myocardial fibrosis, and reduce the incidence of AF [60-62]. Therefore, TGF- $\beta$ is closely related to AF and atrial fibrosis. Our results showed that compared to the CTL group, the levels of TGF- $\beta 1$,type III collagen,P-Smad2/3, and Smad2/3 were significantly higher, and the level of Smad7 was significantly lower in the AF + CTL group.

Association between BRD4 and AF

There are few studies on BRD4 in cardiovascular diseases, especially in AF. It was found that BRD4 played an important role in the pathogenesis of high glucose-induced ventricular fibrosis through the TGF- $\beta$ /SMAD pathway [18], and that inhibition of BRD4 could reduce TGF- $\beta$-induced ventricular fibrosis [19]. Considering that atrial fibrosis and ventricular fibrosis share common pathways, atrial fibrosis and AF have mutual causation, and that the TGF- $\beta$ /SMAD pathway plays an important role in the pathogenesis of atrial fibrosis and $\mathrm{AF}$, we speculated that BRD4 might be involved in the occurrence and development of atrial fibrosis and AF through the TGF- $\beta$ /SMAD pathway. Additionally, at the 40th Annual Meeting of the American Heart Rhythm Society, Professor Yigang Li's team first reported that BRD4 was highly expressed in the left atrial tissue of patients with valvular AF, and found that inhibition of BRD4 expression could attenuate atrial fibrosis and reduce the incidence of $\mathrm{AF}$ [20]. This research indicated, for the first time, that BRD4 inhibitors might be used to prevent and treat atrial fibrosis and AF.

JQ-1 is a specific BRD4 inhibitor that binds to the bromine domain of BET protein with high shape complementarity and nanoscale affinity. This interaction leads to a strong, competitive, and transient replacement of BRD4 from acetylated chromatin, and thereby inhibited signaling events downstream of Pol II, inhibiting the biological effects of BRD4 [63,64]. Therefore, (+)JQ-1 was applied to the SD rats in the AF + JQ1 group in this study.

Our study showed that the mean BRD4 level was significantly higher in participants in the AF group, reAF group, LAV group, and in patients with persistent AF than those in the PSVT group, NreAF group, NLAV group, and patients with paroxysmal AF. Moreover, an elevated level of BRD4 was confirmed in patients with non-valvular and non-ischemic cardiomyopathy AF, suggesting that BRD4 is associated with AF. These results suggest that the BRD4 level is significantly positively correlated with LAVs and postoperative recurrence of $\mathrm{AF}$, and that a high BRD4 level is a predictor of LAVs and postoperative recurrence of AF. The results of this study showed that the mean incidence and duration of AF were significantly higher in 
$\mathrm{SD}$ rats in the $\mathrm{AF}+\mathrm{CTL}$ group than those in the $\mathrm{AF}+\mathrm{JQ1}$ group $(P<0.05)$, suggesting that BRD4 inhibition could reduce the incidence and burden of AF.

Our study showed that the mean collagen volume fraction of the left atrium was significantly higher in SD rats in the AF + CTL group than those in the AF + JQ1 and CTL groups. The mean collagen volume fraction of the left atrium was significantly higher in SD rats in the AF + JQ1 group than that in the CTL group. The area of fibrotic collagen, which was stained blue, was largest in the AF + CTL group, smallest in the CTL group, and second largest in the AF + JQ1 group. These results suggest that BRD4 inhibition could attenuate the degree of left atrial fibrosis. Our study showed that the mean values of BRD4, TGF- $\beta 1$, P-Smad2/3, Smad2/3, and type III collagen were significantly higher in SD rats in the AF + CTL group than those in the AF + JQ1 and CTL groups. The mean values of Smad7 and CX43 were significantly lower among SD rats in the AF + CTL group than those in the AF + JQ1 and CTL groups. These results suggest that inhibition of BRD4 expression could reduce the expression of atrial fibrosis-related proteins. BRD4 may be involved in atrial fibrosis and remodeling through the TGF- $\beta 1 /$ Smad signaling pathway, and thus participate in the occurrence and maintenance of $\mathrm{AF}$.

\section{Strengths and limitations}

This study has several strengths. First, this is the first clinical study to investigate the expression of BRD4 in patients with non-valvular and non-ischemic cardiomyopathy $\mathrm{AF}$, as well as to investigate the relationship between BRD4 and AF recurrence after ablation. Second, this is the first study to investigate the role of BRD4 in atrial fibrosis and AF via the TGF- $\beta$ /Smads signaling pathway. Third, this study is the first to describe the relationship between BRD4 and LAAPD, LVEF, and LVAs.

This study also has several limitations. First, the number of study cases was relatively small, and a large sample of data was needed for observation and analysis. Second, more accurate indicators of left atrial size, such as left atrial volume index, are needed for further validation. Third, the level of BRD4 in peripheral venous blood does not fully represent the level of BRD4 in left atrial tissue, and it is necessary to collect left atrial blood samples and left atrial tissue of patients with AF for further verification (e.g., through myocardial biopsy technology). Fourth, the animal experimental methodology was relatively simple, and the mechanism study was not sufficiently in-depth. BRD4 gene knockout and transfection technology should be employed in the future to enhance BRD4 expression and verify the results of this study from multiple perspectives. Fifth, we did not use enough indicators to reflect atrial fibrosis, and more indicators are needed for further verification (such as ST2 and galectin 3). Sixth, further studies are needed to verify whether BRD4 inhibition could attenuate the degree of LAVs in patients with AF and reduce the postoperative recurrence rate of $\mathrm{AF}$.

\section{Conclusion}

Elevated levels of BRD4 may be used to predict the enlargement and fibrosis of the left atrium, postoperative recurrence of AF after ablation, and reduced LVEF in patients with NVAF. Moreover, the postoperative recurrence rate after ablation of AF was significantly positively correlated with LAAPD and the low voltage regions of the left atrium, and negatively correlated with LVEF and LAA-PEV. Inhibition of BRD4 expression significantly improved the degree of left atrial fibrosis in rats with AF, and reduced the incidence and duration of AF. BRD4 may be involved in atrial fibrosis and remodeling through the TGF- $\beta 1 /$ Smad signaling pathway, thus participating in the occurrence and maintenance of $\mathrm{AF}$.

\section{Funding}

This research did not receive any specific grant from funding agencies in the public, commercial, or not-forprofit sectors. This study was supported by the Affiliated Hospital of Guizhou Medical University (Contract gzwjkj2020-2-001, Grants H-2020-40 from the Guizhou Provincial Health Commission).

\section{References}


[1] Benjamin EJ,Muntner P,Alonso A,Bittencourt MS,Callaway CW,et al.Heart disease and stroke statistics2019 update: a report from the American Heart Association[J].Circulation,2019;139:e56-e528.

[2] Zhang S.Atrial fibrillation in mainland China:epidemiology and current management[J].Heart,2009,95(13):1052-1055.

[3] Chugh SS,Havmoeller R,Narayanan K,Singh D,Rienstra M,et al.Worldwide epidemiology of atrial fibrillation: a Global Burden of Disease 2010 Study[J].Circulation,2014;129:837-847.

[4] Colilla S,Crow A,Petkun W,Singer DE,Simon T,Liu X.Estimates of current and future incidence and prevalence of atrial fibrillation in the US adult population[J].Am J Cardiol,2013;112:1142-1147.

[5] Krijthe BP,Kunst A,Benjamin EJ,Lip GY,Franco OH,Hofman A,Witteman JC,Stricker B-H,Heeringa J.Projections on the number of individuals with atrial fibrillation in the Europ-ean Union, from 2000 to 2060[J].Eur Heart J,2013;34:2746-2751.

[6] Staerk L,Sherer JA,Ko D,Benjamin EJ,Helm RH.Atrial fibrillation: epidemiology,pathop-hysiology, and clinical outcomes[J].Circ Res,2017;120:1501-1517.

[7] Chen C, Zhou X, Zhu M, Chen S, Chen J, Cai H, Dai J, Xu X, Mao W. Catheter ablation versus medical therapy for patients with persistent atrial fibrillation: a systematic review and meta analysis of evidence from randomized controlled trials[J]. J Interv Card El-ectrophysiol 2018;52:9-18.

[8] Packer DL, Mark DB, Robb RA, Monahan KH, Bahnson TD, et al. Effect of catheter ablation vs antiarrhythmic drug therapy on mortality, stroke, bleeding, and cardiac arrest among patients with atrial fibrillation:the CABANA randomized clinical trial[J]. JAMA 2019;321:1261-1274.

[9] Noseworthy PA, Gersh BJ, Kent DM, Piccini JP, Packer DL, Shah ND, Yao X.Atrial fibrillation ablation in practice: assessing CABANA generalizability[J]. Eur Heart J 2019;40:1257-1264.

[10] Blomström-Lundqvist C, Gizurarson S, Schwieler J, Jensen SM, Bergfeldt L, Kennebä-ck G, Rubulis A, Malmborg H, Raatikainen P, Lönnerholm S, Höglund N, Mörtsell D. E-ffect of catheter ablation vs antiarrhythmic medication on quality of life in patients with atrial fibrillation: the CAPTAF randomized clinical trial[J].JAMA 2019;321:1059-1068.

[11] January CT, Wann LS, Calkins H, Chen LY, Cigarroa JE, Cleveland JC Jr, Ellinor PT, Ezekowitz MD, Field ME, Furie KL, Heidenreich PA, Murray KT, Shea JB, Tracy CM, Yancy CW. 2019 AHA/ACC/HRS Focused Update of the 2014 AHA/ACC/HRS Guideline for the Management of Patients With Atrial Fibrillation: A Report of the American C-ollege of Cardiology/American Heart Association Task Force on Clinical Practice Guidelin-es and the Heart Rhythm Society in Collaboration With the Society of Thoracic Surgeons[J]. Circulation,2019;140(2):e125-e151.

[12] Hindricks G, Potpara T, Dagres N, Arbelo E, Bax JJ, et al. 2020 ESC Guidelines for the diagnosis and management of atrial fibrillation developed in collaboration with the European Association of CardioThoracic Surgery (EACTS)[J].Eur Heart J,2021;42(5):373-498.

[13] Mujović N, Marinković M, Lenarczyk R, Tilz R, Potpara TS. Catheter Ablation of Atrial Fibrillation: An Overview for Clinicians[J]. Advances in Therapy, 2017, 34(62):1897-1917.

[14] Andrade JG, Wells GA, Deyell MW, Bennett M, Essebag V, et al. Cryoablation or Drug Therapy for Initial Treatment of Atrial Fibrillation[J]. N Engl J Med,2021,384(4):305-315.

[15] Kawaji T, Shizuta S, Aizawa T, Yamagami S, Kato M, Yokomatsu T, Miki S, Ono K, Kimura T. Impact of catheter ablation for atrial fibrillation on cardiac disorders in patients with coexisting heart failure[J]. ESC Heart Fail,2021,8(1):670-679.

[16] Xu G, Cai J, Liu Z, Liu E, Jing X, Liu T, Zhang Q, Ye L, Li G. Clinical efficacy of "ICE-FIRE" ablation for non-paroxysmal atrial fibrillation[J]. J Interv Card Electrophys-iol,2021,60(2):205-211. 
[17] Sun Y, Xie Y, Du L, Sun J, Liu Z.Inhibition of BRD4 Attenuates Cardiomyocyte Apoptosis via NF- $x$ B pathway in a Rat Model of Myocardial Infarction[J].Cardiovascular Therapeutics, 2018:36(2).

[18] Wang Q, Sun Y, Li T, Liu L, Zhao Y, Li L, Zhang L, Meng Y. Function of BRD4 in the pathogenesis of high glucose-induced cardiac hypertrophy[J]. Mol Med Rep. 2019;19(1):499-507.

[19] Song S, Liu L, Yu Y, Zhang R, Li Y, Cao W, Xiao Y, Fang G, Li Z, Wang X, Wang Q, Zhao X, Chen L, Wang Y, Wang Q. Inhibition of BRD4 attenuates transverse aortic constriction and TGF- $\beta$-induced endothelial-mesenchymal transition and cardiac fibrosis[J]. J Mol Cell Cardiol. 2019;127:83-96.

[20] Shuai Song, Wei Cao, Guojian Fang, et al. Brd4 As A Novel Therapeutic Target For Atrial Fibrosis And Atrial Fibrillation[R]. Heart Rhythm Society Annual Sci-entific Sessions; May 8-11, 2019; San Francisco.

[21] Yang YM, Shi RH, Xu CX, Li L. BRD4 expression in patients with essential hypertension and its effect on blood pressure in spontaneously hypertensive rats. J Am Soc Hypertens. 2018 Dec;12(12):e107-e117.

[22] Cheng C, Liu H, Tan C, Tong D, Zhao Y, Liu X, Si W, Wang L, Liang L, Li J, et al.(2019) Mutation in NPPA causes atrial fibrillation by activating inflammation and cardiac fibrosis in a knock-in rat model. FASEB J 33:8878-8891.

[23] Wu Q, Liu H, Liao J, Zhao N, Tse G, Han B, Chen L, Huang Z, Du Y. Colchicine prevents atrial fibrillation promotion by inhibiting IL-1 $\beta$-induced IL-6 release and atrial fibrosis in the rat sterile pericarditis model. Biomed Pharmacother. 2020 Sep;129:110384. doi: 10.1016/j.biopha.2020.110384.

[24] Zhang T, Wu Y, Hu Z, Xing W, Kun LV, Wang D, Hu N. Small-Molecule Integrated Stress Response Inhibitor Reduces Susceptibility to Postinfarct Atrial Fibrillation in Rats via the Inhibition of Integrated Stress Responses. J Pharmacol Exp Ther. 2021 Sep;378(3):197-206. doi: 10.1124/jpet.121.000491.

[25] Platonov PG, Mitrofanova LB, Orshanskaya V, Ho SY.Structural abnormalities in atrial walls are associated with presence and persistency of atrial fibrillation but not with age[J]. J Am Coll Cardiol. 2011;58(21):2225-2232.

[26] Floria M, Radu S, Gosav EM, Cozma D, Mitu O, Ouatu A, Tanase DM, Scripcariu V, Serban LI. Left Atrial Structural Remodelling in Non-Valvular Atrial Fibrillation: What Have We Learnt from CMR? [J].Diagnostics (Basel),2020;10(3):137.

[27] Jeong joo Woo, Dana C Peters, John V Wylie, et al.Correlation of Left Atrial Voltage Maps with Cardiovascular MR Provides Evidence of Preexistent Scar in Atrial Fibrillation Patients[J].Circulation, 2008,118:771-776.

[28] Canciello G, de Simone G, Izzo R, Giamundo A, Pacelli F, Mancusi C, Galderisi M, Trimarco B, Losi MA. Validation of Left Atrial Volume Estimation by Left Atrial Diameter from the Parasternal Long-Axis View[J]. J Am Soc Echocardiogr,2017;30(3):262-269.

[29] Bossard M, Knecht S, Aeschbacher S, Buechel RR, Hochgruber T, Zimmermann AJ, Kessel-Schaefer A, Stephan FP, Völlmin G, Pradella M, Sticherling C, Osswald S, Kaufma-nn BA, Conen D, Kühne M. Conventional versus 3-D Echocardiography to Predict Arrhythmia Recurrence After Atrial Fibrillation Ablation[J]. J Cardiovasc Electrophysiol,2017;28(6):651-658.

[30] Liao YC, Liao JN, Lo LW, Lin YJ, Chang SL, et al. Left Atrial Size and Left Ventricular End-Systolic Dimension Predict the Progression of Paroxysmal Atrial Fibrillation After Catheter Ablation[J]. J Cardiovasc Electrophysiol,2017;28(1):23-30.

[31] Njoku A, Kannabhiran M, Arora R, Reddy P, Gopinathannair R, Lakkireddy D, Dom-inic P. Left atrial volume predicts atrial fibrillation recurrence after radiofrequency ablation: a meta-analysis[J]. Europace,2018;20(1):33-42.

[32] Di Biase L, Burkhardt JD, Mohanty P, Sanchez J, Mohanty S, et al. Left atrial appendage: an underrecognized trigger site of atrial fibrillation[J]. Circulation,2010;122(2):109-118. 
[33] Kim MN, Lee JJ, Kim SA, Kim YH, Choi JI, Park SM, Park SW, Kim YH, Shim WJ. The difference of predictors for recurrence after catheter ablation of non-paroxysmal atrial fibrillation according to follow-up period[J]. Int Heart J,2014;55(4):312-318.

[34] Shiozawa T, Shimada K, Sekita G, Hayashi H, Tabuchi H, Miura S, Fujimoto S, Ka-doguchi T, Ouchi S, Aikawa T, Al Shahi H, Takahashi S, Miyazaki T, Sumiyoshi M, Na-kazato Y, Daida H. Left Atrial Appendage Volume and Plasma Docosahexaenoic Acid Le-vels Are Associated With Atrial Fibrillation Recurrence After Catheter Ablation[J]. Cardiol Res,2017;8(3):96-104.

[35] Pinto Teixeira P, Martins Oliveira M, Ramos R, Rio P, Silva Cunha P, Delgado AS, Pimenta R, Cruz Ferreira R. Left atrial appendage volume as a new predictor of atrial fibrillation recurrence after catheter ablation[J]. J Interv Card Electrophysiol,2017;49(2):165-171.

[36] Tian X, Zhang XJ, Yuan YF, Li CY, Zhou LX, Gao BL. Morphological and functionnal parameters of left atrial appendage play a greater role in atrial fibrillation relapse aft-er radiofrequency ablation $[\mathrm{J}]$. Sci Rep,2020;10(1):8072.

[37] He Y, Zhang B, Zhu F, Hu Z, Zhong J, Zhu W. Transesophageal echocardiography measures left atrial appendage volume and function and predicts recurrence of paroxysmal atrial fibrillation after radiofrequency catheter ablation[J]. Echocardiography,2018;35(7):985-990.

[38] Polyakova V, Miyagawa S, Szalay Z, Risteli J, Kostin S.Atrial extracellular matrix remodelling in patients with atrial fibrillation[J]. J Cell Mol Med,2008;12:189-208.

[39] Rog-Zielinska EA, Norris RA, Kohl P, Markwald R.The living scar-cardiac fibroblasts and the injured heart[J]. Trends Mol Med, 2016;22: 99-114.

[40] Kohl P and Gourdie RG.Fibroblast-myocyte electrotonic coupling: Does it occur in native cardiac tissue?[J]. J Mol Cell Cardiol, 2014;70: 37-46.

[41] Ongstad E and Kohl P.Fibroblast-myocyte coupling in the heart:Potential relevance for therapeutic interventions[J]. J Mol Cell Cardiol, 2016;91: 238-246.

[42] Nguyen TP, Qu Z and Weiss JN.Cardiac fibrosis and arrhythmogenesis: The road to repair is paved with perils[J]. J Mol Cell Cardiol, 2014;70: 83-91.

[43] Krul SP, Berger WR, Smit NW, van Amersfoorth SC, Driessen AH, van Boven WJ, Fiolet JW, van Ginneken AC, van der Wal AC, de Bakker JM, Coronel R, de Groot JR. Atrial fibrosis and conduction slowing in the left atrial appendage of patients undergoing thoracoscopic surgical pulmonary vein isolation for atrial fibrillation[J].Circ Arrhythm Electrophysiol, 2015;8: 288-295.

[44] Nattel S. Electrical coupling between cardiomyocytes and fibroblasts: Experimental testing of a challenging and important concept[J]. Cardiovasc Res, 2018;114: 349-352.

[45] Pellman J and Sheikh F.Atrial fibrillation: Mechanisms, therapeutics, and future directi-ons[J]. Compr Physiol,2015;5:649-665.

[46] Siebermair J,Kholmovski EG,Marrouche N.Assessment of left atrial fibrosis by late gadolinium enhancement magnetic resonance imaging[J].JACC Clin Electrophysiol,2017; 3: 791-802.

[47] Zghaib T, Keramati A, Chrispin J, Huang D, Balouch MA, Ciuffo L, Berger RD, M-arine JE, Ashikaga H, Calkins H, Nazarian S, Spragg DD.Multimodal examination of atrial fibrillation substrate[J].JACC Clin Electrophysiol,2018; 4: 59-68.

[48] Floria M, Radu S, Gosav EM, Cozma D, Mitu O, Ouatu A, Tanase DM, Scripcariu V, Serban LI. Left Atrial Structural Remodelling in Non-Valvular Atrial Fibrillation: What Have We Learnt from CMR? [J].Diagnostics (Basel),2020;10(3):137. 
[49] Chen J, Arentz T, Cochet H, Muller-Edenborn B, Kim S, Moreno-Weidmann Z, Min-ners J, Kohl P, Lehrmann H, Allgeier J, Trenk D, Hocini M, Jais P, Haissaguerre M, Jad-idi A. Extent and spatial distribution of left atrial arrhythmogenic sites, late gadolinium enhancement at magnetic resonance imaging, and low-voltage areas in patients with persistent atrial fibrillation: comparison of imaging vs. electrical parameters of fibrosis and arrhythmogenesis[J]. Europace,2019;21(10):1484-1493.

[50] Qureshi NA, Kim SJ, Cantwell CD, Afonso VX, Bai W, et al. Voltage during atrial fibrillation is superior to voltage during sinus rhythm in localizing areas of delayed enhancement on magnetic resonance imaging: an assessment of the posterior left atrium in patients with persistent atrial fibrillation[J]. Heart Rhythm,2019;16:1357-1367.

[51] Jeong joo Woo, Dana C Peters, John V Wylie, et al.Correlation of Left Atrial Voltage Maps with Cardiovascular MR Provides Evidence of Preexistent Scar in Atrial Fibrillation Patients[J].Circulation, 2008,118:771-776.

[52] Ling LH, McLellan AJ, Taylor AJ, Iles LM, Ellims AH, Kumar S, Teh A, Lee G, Wong MC, Azzopardi S, Sellenger MA, Morton JB, Kalman JM, Kistler PM. Magnetic resonance postcontrast T1 mapping in the human atrium: validation and impact on clinical outcome after catheter ablation for atrial fibrillation[J]. Heart Rhythm,2014;11(9):1551-1559.

[53] Lee J, Thornhill RE, Nery P, Robert deKemp, Pena E, Birnie D, Adler A, Ukwatta E. Left atrial imaging and registration of fibrosis with conduction voltages using LGE-MRI and electroanatomical mapping[J]. Computers in Biology and Medicine, 2019, 111:103341.

[54] Yagishita A, Sparano D, Cakulev I, Gimbel JR, Phelan T, Mustafa H, De Oliveira S, Mackall J, Arruda M. Identification and electrophysiological characterization of early left atrial structural remodeling as a predictor for atrial fibrillation recurrence after pulmonary vein isolation[J]. J Cardiovasc Electrophysiol,2017;28(6):642650.

[55] Begg GA, Karim R, Oesterlein T, Graham LN, Hogarth AJ, Page SP, Pepper CB, Rhode K, Lip GYH, Holden AV, Plein S, Tayebjee MH. Left atrial voltage, circulating biomarkers of fibrosis, and atrial fibrillation ablation[J]. PLoS One,2018;13(1):e0189936.

[56] Masuda M, Fujita M, Iida O, Okamoto S, Ishihara T, Nanto K, Kanda T, Tsujimura T, Matsuda Y, Okuno S, Ohashi T, Tsuji A, Mano T. Left atrial low-voltage areas predict atrial fibrillation recurrence after catheter ablation in patients with paroxysmal atrial fibrillation[J]. Int J Cardiol,2018;257:97-101.

[57] Masuda M, Asai M, Iida O, Okamoto S, Ishihara T, Nanto K, Kanda T, Tsujimura T, Matsuda Y, Okuno S, Hata Y, Mano T. Additional Low-Voltage-Area Ablation in Patients With Paroxysmal Atrial Fibrillation: Results of the Randomized Controlled VOLCANO T-rial[J]. J Am Heart Assoc,2020;9(13):e015927.

[58] Morikawa M, Derynck R, Miyazono K. TGF- $\beta$ and the TGF- $\beta$ Family: Context-Dependent Roles in Cell and Tissue Physiology[J]. Cold Spring Harb Perspect Biol. 2016;8(5):a021873.

[59] Wang Y, Cai H, Li H, Gao Z, Song K. Atrial overexpression of microRNA-27b atenuates angiotensin II-induced atrial fibrosis and fibrillation by targeting ALK5[J]. Hum Cell. 2018;31(3):251-260.

[60] Guo J, Jia F, Jiang Y, Li Q, Yang Y, Xiao M, Xiao H. Potential role of MG53 in the regulation of transforming-growth-factor- $\beta 1$-induced atrial fibrosis and vulnerability to atrial fibrillation[J]. Exp Cell Res. 2018;362(2):436-443.

[61] Shen H, Wang J, Min J, Xi W, Gao Y, Yin L, Yu Y, Liu K, Xiao J, Zhang YF, Wang ZN. Activation of TGF- $\beta 1 / \alpha-S M A / C o l$ I Profibrotic Pathway in Fibroblasts by Galectin-3 Contributes to Atrial Fibrosis in Experimental Models and Patients[J]. Cell Physiol Biochem. 2018;47(2):851-863.

[62] Cao F, Li Z, Ding WM, Yan L, Zhao QY. LncRNA PVT1 regulates atrial fibrosis via miR-128-3p-SP1TGF- $\beta 1-S m a d$ axis in atrial fibrillation[J]. Molecular Medicine, 2019, 25(1):7. 
[63] Mumby S, Gambaryan N, Meng C, Perros F, Humbert M,Wort SJ,Adcock IM.Bromo-domain and extraterminal protein mimic JQ1 decreases inflammation in human vascular endothelial cells: Implications for pulmonary arterial hypertension[J].Respirology,2017;22:157-164.

[64] Mio C, Conzatti K, Baldan F, Allegri L, Sponziello M,Rosignolo F, Russo D, Filetti S,Damante G.BET bromodomain inhibitor JQ1 modulates microRNA expression in thyroid cancer cells[J]. Oncol Rep,2018;39:582-588.

\section{Hosted file}

Figures.doc available at https://authorea.com/users/457157/articles/554011-expression-ofbrd4-in-patients-with-nonvalvular-atrial-fibrillation-and-its-role-in-postoperativerecurrence-and-the-development-of-atrial-fibrosis

\section{Hosted file}

tables.doc available at https://authorea.com/users/457157/articles/554011-expression-ofbrd4-in-patients-with-nonvalvular-atrial-fibrillation-and-its-role-in-postoperativerecurrence-and-the-development-of-atrial-fibrosis 